\section{ZERTIFIZIERTE FORTBILDUNG}

IN ZUSAMMENARBEIT MIT DER BAYERISCHEN LANDESÄRZTEKAMMER

Folge 130

Hier können Sie CME-Punkte sammeln, und zwar a) für die Pflichtfortbildung aller Vertragsärzte und b) für freiwillige Fortbildungszertifikate, die viele Landesärztekammern anbieten.

Die folgenden Multiple-Choice-Fragen beziehen sich thematisch auf den vorangegangenen MMW-

Fortbildungsbeitrag auf den Seiten 36-39. Hinweis: Die Antworten auf die CME-Fragen ergeben sich nur zum Teil direkt aus dem Text. Einige Fragen beruhen auf medizinischem Basiswissen!

Wenn Sie 70\% der Fragen richtig beantworten, erhalten Sie 2 CME-Punkte.

Beantworten Sie $100 \%$ korrekt, erhalten Sie 3 CME-Punkte.

Einsendeschluss für die Teilnahme im Internet (www.cme-punkt.de): 14. September 2007

Einsendeschluss für die Teilnahme per Brief: 28. Juni 2007
MMW

Fortschritte der Medizin

CME-Herausgeber- und Review-Board:

Dr. H. J. K. Barwitz, Prof. Dr. A. Berghaus, Prof. Dr.

Dr. h.c. Th. Brandt, Prof. Dr. W. G. Daniel, Prof. Dr.

W. Eisenmenger, Prof. Dr. K. Friese, Prof. Dr. H.

S. Füessl, Prof. Dr. B. Göke, Prof. Dr. R. Gradinger, Prof. Dr. Dr. h.c. A. Hofstetter, Prof. Dr. H. Holzgreve, Prof. Dr. A. Imdahl, Prof. Dr. K. Krüger, Prof. Dr. H.-J. Möller, Prof. Dr. Dr. h.c. G. Plewig, Prof. Dr. D. Reinhardt, U. Weigeldt.

\title{
Fragen zum Thema „Fersenschmerz“
}

\section{- 1. In welche zwei großen Gruppen wird der Fersenschmerz unterschieden? \\ A Hinterer und vorderer Schmerz \\ B Oberer und plantarer Fersenschmerz \\ C Medialer und lateraler Schmerz \\ D Oberer und ventraler Schmerz \\ E Plantarer und subkalkanearer Schmerz}

\section{Bei wie viel Prozent der beschwerde- freien Allgemeinbevölkerung gelingt der Nachweis eines Fersensporns?}
A ca. $50 \%$
B ca. $70 \%$
C ca. $3 \%$
D ca. $10 \%$
E ca. $98 \%$

\section{A immer operativ. \\ B meist konservativ. \\ C nie konservativ. \\ D nie operativ. \\ E nur chirurgisch sinnvoll.}

- 3. Die Therapie der Haglund-Exostose ist

\section{- 4. Die Apophysitis calcanei}

A kann durch eine Röntgenaufnahme diagnostiziert werden.

B kann nie durch eine Röntgenaufnahme diagnostiziert werden.

C tritt nur beim Erwachsenen auf.

D muss immer operiert werden.

E dauert meist über Jahre an.
- 5. Beim plantaren Fersenschmerz kann der Fersensporn

A nie diagnostiziert werden.

B in ca. 90\% der Fälle diagnostiziert werden.

C nur bei Kindern diagnostiziert werden.

D immer diagnostiziert werden.

E in ca. 50\% der Fälle diagnostiziert werden.

\section{Zum Ankerwinden-Mechanismus} gehört nicht

A das Anspannen der Plantarfaszie.

B die Verstärkung der Längswölbung des Fußes.

C die Mittelfußadduktion.

D die Rückfußinversion.

E die Außenrotation des Beines.

7. Der hintere Kalkaneusneigungswinkel

A wird als CCD-Winkel bezeichnet.

B ist in der Regel kleiner als $32^{\circ}$.

C ist bei allen Menschen nahezu identisch.

D wird auch als Fowler-Philip-Winkel bezeichnet.

E wird durch die klinische Untersuchung bestimmt.

\section{- 8. Die benigne Knochenzyste des Kalkaneus}

A ist die häufigste Tumorerkrankung des Fersenbeins.

B tritt gehäuft bilateral auf.

C muss fast immer operiert werden.

D neigt zur malignen Entartung.

E verursacht nie Komplikationen.

- 9. Die Stressfraktur des Kalkaneus

A kann nur operativ behandelt werden.

B ist nahezu immer nativradiologisch zu diagnostizieren.

C kann im MRT nur sehr schlecht dargestellt werden.

D gehört zu den Tumorerkrankungen des Fußes.

E entsteht durch wiederholte und ungewohnte Belastungen des Fersenbeins.

\section{- 10. Der plantare Fersenschmerz kann}

A konservativ nicht behandelt werden.

B nicht operativ therapiert werden.

C in der Regel konservativ gut behandelt werden.

D nur durch Injektionen erfolgreich therapiert werden.

E durch physikalische Therapie nicht beeinflusst werden.

\section{ANTWORTFORMULAR}

Ich versichere, alle Fragen ohne fremde Hilfe beantwortet zu haben.

Mit dem Einreichen dieses Fragebogens erklärt sich der Einreichende damit einverstanden, dass die angegebenen Daten zum Zweck der Teilnahmebestätigung gespeichert und bei erfolgreicher Teilnahme auch an den Einheitlichen Informationsverteiler (EIV) der Ärztekammern weitergegeben werden.

Wichtiger Hinweis:

Eine Auswertung ist künftig nur noch möglich, wenn Sie Ihre EFN auf dem Antwortformular angeben! Nähere Hinweise hierzu unter: www.cme-punkt.de/faq.htm

\section{„Fersenschmerz“}

MMW-Fortschr. Med. Nr. 24 / 2007 (149. Jg.)

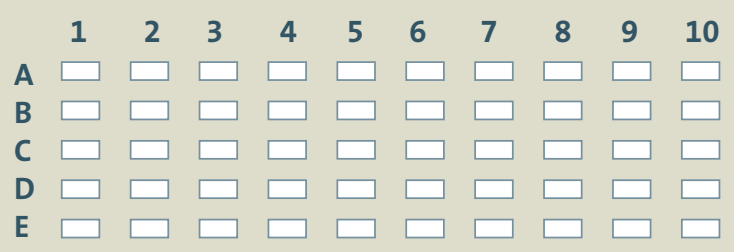

Bitte kreuzen Sie pro Frage je eine Antwort deutlich an.
Und so kommen Sie zu Ihren Punkten:

Teilnahme im Internet: unter www.cme-punkt.de. Dort führen wir für Sie ein elektronisches Punktekonto.

Teilnahme per Brief Fragebogen ausfüllen und mit einem frankierten Rückumschlag an: Urban \& Vogel Medien und Medizin Verlagsgesellschaft, Redaktion MMW-Fortschritte de Medizin, Abt. Fortbildungszertifikat, Neumarkter Str. 43 81673 München. 